BONPLANDIA 18(1): 39-44. 2009

\title{
CARACTERIZACIÓN DE HYBANTHUS LEUCOPOGON (VIOLACEAE), UNA ESPECIE ENDÉMICA DE CORRIENTES
}

\author{
MICAELA NOEMÍ SEO ${ }^{1,2}$
}

\begin{abstract}
Summary: Seo, M. N. 2009. Characterization of Hybanthus leucopogon (Violaceae), an endemic species of Corrientes. Bonplandia 18(1): 39-44. ISSN: 0524-0476.

The macro and micromorphological characterization of Hybanthus leucopogon Sparre were realized, in order to improve the taxonomic knowledge related to the Flora of Argentina, which has been in Hybanthus still poorly studied. The micromorphological patterns of the seed coat and the foliar margin of this species were analyzed, and these observations have revealed the presence of a pattern of specific micro-ornamentation on the seed coat and the foliar surface, which would be used in the infragenerical identification of Hybanthus.
\end{abstract}

Key words: Argentina, foliar margin, micromorphology, seed coat.

Resumen: Seo, M. N. 2009. Caracterización de Hybanthus leucopogon (Violaceae), una especie endémica de Corrientes. Bonplandia 18(1): 39-44. ISSN: 0524-0476.

Se realizó la caracterización macro y micromorfológica de Hybanthus leucopogon Sparre, aspecto aún muy poco estudiado en Hybanthus. Se analizaron los patrones micromorfológicos de la superficie seminal y del margen foliar de esta especie; estas observaciones han revelado la presencia de un patrón específico de micro-ornamentación en la cubierta seminal y la superficie foliar que podrían ser utilizados en la identificación infragenérica de Hybanthus.

Palabras clave: Argentina, cubierta seminal, margen foliar, micromorfología.

\section{Introducción}

El género Hybanthus Jacq. está conformado por más de 100 especies distribuidas en regiones tropicales y subtropicales del Viejo y Nuevo Mundo (Todzia, 1989). Hekking (1988) en su revisión de Violaceae ha citado más de 70 especies para el continente americano, cerca de 10 especies en Madagascar, 5 en Asia y 10 en Australia aproximadamente.
En Argentina habitan 16 especies nativas en el norte y centro del país (Sparre, 1950; Sanso \& al., 2008). Una de ellas, Hybanthus leucopogon, ha sido descripta por Sparre (1950) y desde entonces no existen datos referidos a la misma, excepto las citas en los Catálogos de Plantas Vasculares (Xifreda \& Sanso, 1999; Sanso \& al., 2008). Esta especie es endémica de la provincia de Corrientes, donde ha sido coleccionada ocasionalmente, sin embargo el conocimiento taxonómico referi-

${ }^{1}$ Becaria Postdoctoral CONICET-IFEVA, Facultad de Agronomía, Universidad de Buenos Aires. Av. San Martín 4453, C1417DSE Ciudad de Buenos Aires, Argentina.

${ }^{2}$ Laboratorio de Citogenética y Evolución, Facultad de Ciencias Exactas y Naturales, Universidad de Buenos Aires. E-mail: micaseo@ege.fcen.uba.ar 
do a esta especie es aún escaso. La revisión realizada en las especies de Hybanthus en Argentina (Seo, 2008) ha permitido ampliar el conocimiento sistemático referido a este género.

El objetivo del presente trabajo es realizar una caracterización de Hybanthus leucopogon a nivel macro y micromorfológico, con el análisis de caracteres adicionales que podrían ser útiles en la determinación específica del género Hybanthus.

\section{Materiales y métodos}

Se han revisado los ejemplares de Hybanthus leucopogon depositados en los principales herbarios de Argentina: BAA, BAB, BAF, CTES, LIL, LP y SI. Se ha realizado una breve caracterización macro y micro-morfológica; también se detalla la distribución geográfica y la fenología de esta entidad.

Los análisis micromorfológicos se efectuaron sobre semillas y hojas frescas o provenientes de ejemplares de herbario que se montaron sobre taquitos metálicos cubiertos con cinta doble faz. Las muestras vegetales se metalizaron con oro-paladio mediante un equipo JEOL JPC-1100, se observaron al Microscopio Electrónico de Barrido (MEB) y se fotografiaron digitalmente. Para ello se utilizaron dos equipos: JEOL JSM- T 100 y JEOL JSM-6360 LV, ambos pertenecientes al Museo de Ciencias Naturales de La Plata. Se observaron al menos 5 semillas y 5 hojas provenientes de distintos ejemplares de herbario.

En los estudios micromorfológicos realizados en semilla se analizaron distintos patrones de distribución celular, caracteres de la microesculturación primaria y secundaria de la superficie seminal. Para la descripción de los caracteres micro-morfológicos se consideraron los trabajos de Barthlott (1981, 1984).

En los estudios de la micromorfología foliar se analizó la presencia y morfología de pelos y papilas sobre la superficie foliar y características micromorfológicas del borde de las hojas. La descripción de los caracteres de los tricomas y glándulas se realizó de acuerdo a las clasificaciones y definiciones establecidas por Lawrence (1967) y Theobald \& al. (1979).

\section{Resultados}

\section{Caracterización morfológica de Hybanthus leucopogon Sparre}

Fig. 1

Sparre, B., Lilloa 23: 562. 1950. Typus: Argentina. Corrientes: Dep. Mercedes, Justino Solari, 8X-1942, Ibarrola s.n. (holotypus LP!, isotypus LIL!).

Iconografía: Sparre, Lilloa 23: 565, fig. 1-10; fig. 3 A-G. 1950

Hierba pequeña de 30 a $50 \mathrm{~cm}$ de altura, simple o apenas ramificada en la base. Hojas en general opuestas, glabras, angostamente lanceoladas, elípticas a subovadas, con breve pecíolo de 2-4 mm de long, de margen entero, subentero o lobado, la base atenuada y el ápice acuminado, de 30-60 x 18-28 mm. La hojas superiores pueden presentar una disposición alterna, son más pequeñas que las inferiores, de forma lanceolada a acuminada. Flores lilas a violáceas dispuestas en pseudo-racimo terminal. Sépalos subiguales, lanceolados de 23,4 mm de long. Pétalos superiores oblongos a ovoides, de 2-3,4 $\mathrm{mm}$ de long. Pétalos laterales subfalcados, oblongos, con ala lateral y contraídos en la región media, de 3-6 mm de long. Labelo unguiculado, hasta $18 \mathrm{~mm}$ de long, con la base angosta y la lámina de tipo suborbicular-rectangular, notablemente extendida, con el extremo en punta, en general más ancha que larga, hasta $15 \mathrm{~mm}$ de ancho, $\mathrm{y}$ mácula central en la base, blanca y violeta. Estambres 5, desiguales, subsésiles de 2-3 $\mathrm{mm}$ de long, los 2 inferiores con el dorso piloso y con una apéndice nectarífero en forma de verruga pequeña. Ovario glabro y globoso y estilo con el ápice dilatado. Cápsula trígona- subovada, de 10-12 mm de diám., las semillas son ovoides con la calaza engrosada (Fig. 2 A) y se diferencia en la zona de la 
M. N. Seo, Caracterización de Hybanthus leucopogon (Violaceae)

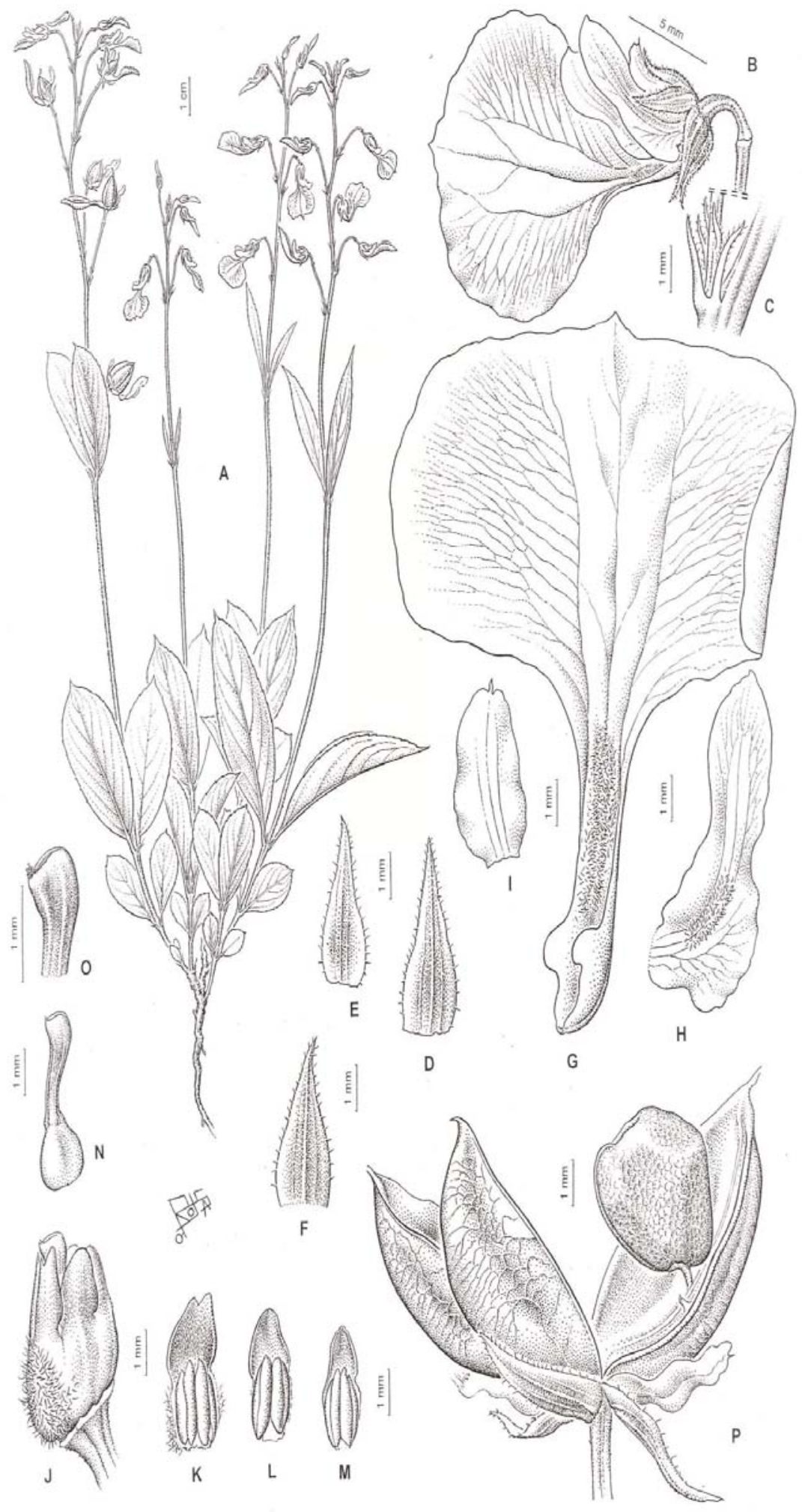

Fig. 1. Hybanthus leucopogon. A: aspecto general. B: flor. C: pecíolo. D: sépalo inferior. E: sépalo lateral. F: sépalo superior. G: labelo. H: pétalo lateral. I: pétalo superior. J: estambres y estilo. K: estambre inferior. L: estambre lateral. M: estambre superior. N: gineceo. O: estigma. P: fruto con semilla (A-P, Nicora 5780). 
micrópila un arilo notorio o elaiosoma, de 23,5 mm de long. $n=16$ (Seo, 2008).

Distribución geográfica y hábitat: Especie endémica de Argentina, donde crece en áreas restringidas de la provincia de Corrientes, en los departamentos de Mercedes, Monte Caseros, Curuzú Cuatiá y Berón de Astrada. Se la ha coleccionado sobre suelos desnudos, áridos y rocosos.

Fenología: Florece a partir de los meses de septiembre a enero, y fructifica desde diciembre hasta abril.

\section{Caracterización micromorfológica}

Los estudios micro-morfológicos realizados mediante el MEB han revelado patrones característicos en la microestructura primaria y secundaria de la cubierta seminal y en el margen foliar.

En la micro-esculturación primaria, las células de la cubierta seminal se diferencian como tetra a hexagonales (Fig. 2 B). Las paredes anticlinales del borde celular son notablemente prominentes (Fig. 2 B-C), así como también ciertas regiones de las paredes periclinales sobresalen de la cubierta seminal, $\mathrm{y}$ algunos estomas se diferencian entre las mismas (Fig. 2 C). En relación a la microesculturación secundaria, esta especie presenta una superficie seminal marcadamente estriada (Fig. 2 C), que se superponen a los patrones de la esculturación primaria.

El margen foliar de Hybanthus leucopogon se caracteriza por ser entero a subentero, y en el patrón de micromorfología foliar observado al MEB, se observan de cuatro a cinco hileras de papilas puntiagudas (Fig. 2 D-E). Además se aprecian glándulas globosas (Fig. 2 E-G) ubicadas entre los lóbulos del margen de la hoja. Por otro lado, se diferencian sobre la cara adaxial de la superficie foliar pelos verrucosos (Fig. $2 \mathrm{H}-\mathrm{I}$ ), los mismos exhiben una morfología de tipo arrosetada.

Material examinado: ARGENTINA. Corrientes: Dep. Berón de Astrada: ruta nac. 12 y ayo. Santa María, 04-X-1971, fl, Carnevali 2580 (CTES). Dep. Curuzú-Cuatiá: cerca de Baibiene,
27-XI-1940, fl, Castellanos s. n. (BA). Dep. Mercedes: Mercedes, 6-II-1925, fl, Millán 289 \& 349 (BA); Sur de Mercedes, 23-03-1940, fl, fr, Spegazzini 23 \& 31 (LP); alrededores hacia el Norte, XI-1936, fl, fr, Rodriguez 810 (LP); Justino Solari, 8-X-1942, fr, Ibarrola 2591 (LP); ruta 123, Km 89, 5 Km E de entrada a Yofre, 04-IV-1994, fl, fr, Solis Neffa 48 (CTES)*; $11 \mathrm{Km}$ al S. de Mercedes, antiguo camino a Curuzú-Cuatiá, Pajarito, 23II-1984, fl, Tressens 2442 (CTES); Ruta 119, 28 km S. del acceso a Mercedes, 19-II-1984, fl, Tressens 2208 (CTES); Ruta 12, entre Mercedes y Solari, 08-X-1971, fl, Carnevali 2584 (CTES); about $30 \mathrm{~km}$ E. of Mercedes, on road to Paso de los Libres, 5-XI-1976, fl, fr, Pedersen 11358 (K); Ruta antiguo camino a Curuzá Cuatiá, a 11 km al sur de Mercedes, 12-XII-2006, fl, fr, Seo 34 (BAFC)*; Ea. Villlanueva, $8 \mathrm{~km}$ al sur de Mercedes, 28-II-2008, fl, fr, Seo 58 (BAFC)*. Dep. Monte Caseros: Ea. Tranqueras, cerca de Monte Caseros, 05-XII-1949, fl, Nicora 5780 y 6065 (SI)*; Gral. Avalos: arroyo Curupí, 21-II-1979, fl fr, Schinini 17495 (CTES)*.

Obs.: Sparre (1950) propuso una clasificación infragenérica distinta a la publicada previamente por Schulze-Menz (1936), en la que Hybanthus leucopogon se encuentra incluida dentro del Grupo Oppositifoliae, sin embargo este grupo no ha sido publicado válidamente. Los estudios realizados en otras especies de Hybanthus (Seo, 2008) sugieren que la clasificación sistemática sugerida por Sparre (1950) parece más adecuada debido a la presencia de caracteres más afines con otras especies de hojas opuestas en comparación con taxones de hojas alternas (Grupo Alternifoliae). Estos caracteres no han sido considerados en las secciones establecidas por Schulze-Menz (1936) y los mismos han exhibido importancia a nivel sistemático en la clasificación infragenérica de Hybanthus.

\section{Agradecimientos}

Deseo agradecer especialmente al Dr. M. Dematteis, Alicia López y al IBONE por la

\footnotetext{
* Material utilizado para los estudios micromorfológicos
} 

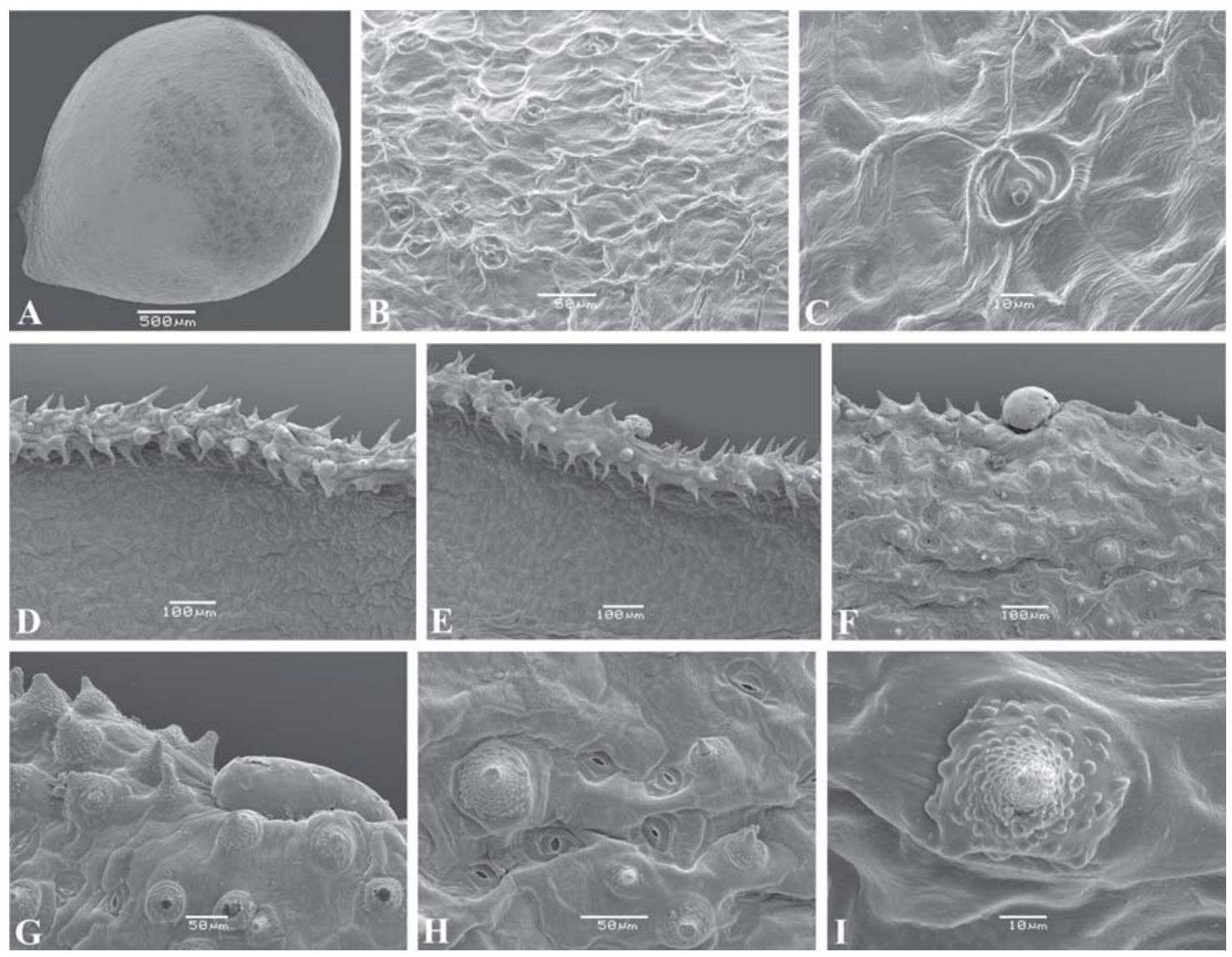

Fig. 2. Fotomicrografías en MEB de H. leucopogon. A: aspecto general de semilla. B: detalle de cubierta seminal. C: detalle de estoma. D: margen foliar, cara abaxial. E: detalle de margen foliar con glándula. F: margen foliar, cara adaxial. G: detalle de glándula. H: detalle de superficie foliar. I: detalle de pelo verrucoso (A-C, Solis Neffa 48; D-I, Seo 34).

ayuda durante los viajes de colección; a los curadores de los herbarios (BAA, BAB, BAF, BAFC, CTES, LP, LIL, MCNS, SI); al Dr. F. Zuloaga, Vladimiro Dudás y Francisco Rojas por la lámina ilustrada, y a Rafael Urrejola por la excelente asistencia en el MEB. Este trabajo se realizó con el apoyo de CONICET, IAPT Research Grants 2007.

\section{Bibliografía}

BARTHLOTT, W. 1981. Epidermal and seed surface characters of plants: systematic applicability and some evolutionary aspects. Nord. J. Bot. 1 (3): 345353.

. 1984. Microstructural features of seed surfaces. En: V. H. Heywood \& D. M. Moore (eds.). Current concepts in plant taxonomy. Systematics association special vol. 25: 95-105. Academic
Press, London.

HEKKING, W. H. A. 1988. Violaceae. Fl. Neotrop. Monogr. 46(1): 1-204.

LAWRENCE, G. H. M. 1967. Taxonomy of the vascular plants. Oxford \& IBH Publishing Co. 761 pp.

SANSO, A. M., M. N. SEO, C. C. XIFREDA \& J. P. SOUZA. 2008. Violaceae. En: F. O. Zuloaga, O. Morrone \& M. Belgrano (eds.). Catálogo de las Plantas Vasculares del Cono Sur. Monogr. Syst. Bot. Missouri Bot. Gard.

SCHULZE-MENZ, G. K. 1936. Morphologischsystematische Studien über die Gattung Hybanthus. Bot. Jahrb. Syst. 67: 437-492.

SEO, M. N. 2008. Estudios sistemáticos y evolutivos en especies argentinas del género Hybanthus. (Violaceae). Tesis Doctoral. Universidad de Buenos Aires, Argentina. 174 pp.

SPARRE, B. 1950. Estudio sobre las Violáceas argentinas I. Los géneros de Hybanthus y Anchietea. Lilloa 23: 515-574.

THEOBALD, W. L., J. L. KRAHULIK \& R. C. 
BONPLANDIA 18(1). 2009

ROLLINS. 1979. Trichome description and classification. En: C. R. Metcalfe \& L. Chalk (eds.). Anatomy of the Dicotyledons. Clarendon Press, Oxford. $2^{\circ}$ ed., vol. 1: 40-53.

TODZIA, C. A. 1989.A nes species of Hybanthus (Violaceae) from Panama. Ann. Missouri Bot.
Gard. 76: 360-362.

XIFREDA, C. C. \& A. M. SANSO. 1999. Violaceae. En: F. Zuloaga \& O. Morrone (eds.). Catálogo de las Plantas Vasculares de la Argentina II. Dicotyledoneae. Monogr. Syst. Bot. Missouri Bot. Gard. 74: 1170-1177, 1246.

Original recibido el 11 de mayo de 2009; aceptado el 30 de junio de 2009. 